\title{
Identification of Hub Diagnostic Biomarkers and Candidate Therapeutic Drugs in Heart Failure
}

\author{
Yang Guo $\mathbb{D}^{1-4}$ \\ Bobin Ning ${ }^{5}$ \\ Qunhui Zhang ${ }^{1-4}$ \\ Jing $\mathrm{Ma}^{4}$ \\ Linlin Zhao ${ }^{1-4}$ \\ QiQin Lu ${ }^{1-4}$ \\ Dejun Zhang ${ }^{1,4}$
}

\begin{abstract}
'Research Center for High Altitude Medicine, Medical College of Qinghai University, Xining, 8I000I, People's Republic of China; ${ }^{2}$ Key Laboratory of Application and Foundation for High Altitude Medicine Research in Qinghai Province, Medical College of Qinghai University, Xining, 8I000I, People's Republic of China; ${ }^{3}$ Qinghai-Utah Joint Research Key Lab for High Altitude Medicine, Medical College of Qinghai University, Xining, 8I000I, People's Republic of China; ${ }^{4}$ Department of EcoEnvironmental Engineering, Qinghai University, Xining, 810016, People's Republic of China; ${ }^{5}$ Department of Medicine, The General Hospital of the People's Liberation Army, Beijing, 100038, People's Republic of China
\end{abstract}

Purpose: The objective of this study was to identify the potential regulatory mechanisms, diagnostic biomarkers, and therapeutic drugs for heart failure (HF).

Methods: Differentially expressed genes (DEGs) between HF and non-failing donors were screened from the GSE57345, GSE5406, and GSE3586 datasets. Database for Annotation Visualization and Integrated Discovery and Metascape were used for Gene Ontology and Kyoto Encyclopedia of Genes and Genomes analyses respectively. The GSE57345 dataset was used for weighted gene co-expression network analysis (WGCNA). The intersecting hub genes from the DEGs and WGCNA were identified and verified with the GSE5406 and GSE3586 datasets. The diagnostic value of the hub genes was calculated through receiver operating characteristic analysis and net reclassification index (NRI). Gene set enrichment analysis (GSEA) was used to filter out the signaling pathways associated with the hub genes. SYBYL 2.1 was used for molecular docking of hub targets and potential HF drugs obtained from the connection map.

Results: Functional annotation of the DEGs showed enrichment of negative regulation of angiogenesis, endoplasmic reticulum stress response, and heart development. PTN, LUM, ISLR, and ASPN were identified as the hub genes of HF. GSEA showed that the key genes were related to the transforming growth factor- $\beta$ (TGF- $\beta$ ) and Wnt signaling pathways. Sirolimus, LY-294002, and wortmannin have been confirmed as potential drugs for HF.

Conclusion: We identified new hub genes and candidate therapeutic drugs for HF, which are potential diagnostic, therapeutic and prognostic targets and warrant further investigation. Keywords: differentially expressed genes, weighted gene co-expression network analysis, diagnostic biomarkers, therapeutic drugs, heart failure

\section{Introduction}

$\mathrm{HF}$ is a clinical syndrome characterized by congestion of the lungs and vena cava, leading to abnormal heart structure or function, which is the final stage of the development of heart disease. ${ }^{1}$ Approximately 40 million people worldwide suffer from $\mathrm{HF}$, and the incidence rates are steadily rising. ${ }^{2}$ Despite significant progress in the HF management in recent decades, the treatment options are mainly palliative rather than curative. ${ }^{3}$ Given the complex pathogenesis of HF, it is essential to elucidate the underlying molecular mechanisms in order to identify potential therapeutic targets and prognostic markers.

Bioinformatics is a high-throughput technique that can screen multiple databases to identify the potential pathological biomarkers of various diseases. ${ }^{4}$ Weighted gene co-expression network analysis (WGCNA) is a systems biology application that mines the genetic interaction networks to construct highly
Correspondence: Dejun Zhang Department of Eco-Environmental Engineering, Qinghai University, Qinghai, 810016, People's Republic of China Email djzhangqhu@163.com 
coordinated gene modules. ${ }^{4}$ WGCNA has been widely used for detecting disease biomarkers, and elucidating biological mechanisms and drug interactions. ${ }^{5-7}$ Although biomarkers of HF have been identified, but due to heterogeneity of HF and its complicated pathophysiological manifestations, a single gene cannot accurately predict the characteristics of HF. ${ }^{8,9}$

In this study, the differentially expressed genes (DEGs) between HF patients and non-failing donors (NFD) were screened from multiple GEO datasets and functionally annotated. The hub genes were then screened through the degree of connectivity in the PPI network, and used to build a co-expression network with WGCNA. The intersecting hub genes between DEGs and WGCNA were identified and validated in other human $\mathrm{HF}$ datasets. Gene set enrichment analysis (GSEA) was used to discover the signaling pathways associated with these hub genes. Finally, the potential HF drugs were predicted through the Connectivity Map (cMap) database, and molecular docking between the drug candidates and hub genes was simulated using SYBYL 2.1 software.

We conducted a bioinformatics analysis using DEGS and WGCNA to further investigate the occurrence and development of $\mathrm{HF}$ and identify potential therapeutic drugs for biomarkers of HF.

\section{Materials and Methods \\ Data Collection and Preprocessing}

The study design is outlined in Figure 1. We searched the GEO database and included data on human heart tissue samples in this study. The mRNA expression profiles from HF and NFD samples were downloaded from the GSE57345, GSE5406 and GSE3586 datasets of the GEO database (http://www.ncbi.nlm.nih.gov/geo/). The subjects included in our study suffered from heart failure with reduced ejection fraction (HFrEF) and heart failure with preserved ejection fraction (HFpEF). Among them were 96 patients with ischemic heart disease, 84 patients with dilated cardiomyopathy (CMP), and 139 non-heart failure samples from GSE57345. ${ }^{10}$ The GSE5406 dataset included 16 samples without heart failure, 86 patients with dilated cardiomyopathy (CMP), and 108 patients with ischemic heart disease. ${ }^{11}$ The GSE3586 dataset included 13 patients with dilated cardiomyopathy and 15 patients without heart failure. ${ }^{12}$ The gene annotation files of GSE57345, GSE5406 and GSE3586 were GPL11532, GPL96, and GPL3050 respectively. GEO2R online tool was used for screening DEGs between HF and NFD with $\mathrm{p}<0.05$ (calculated by $t$-test) as the threshold.

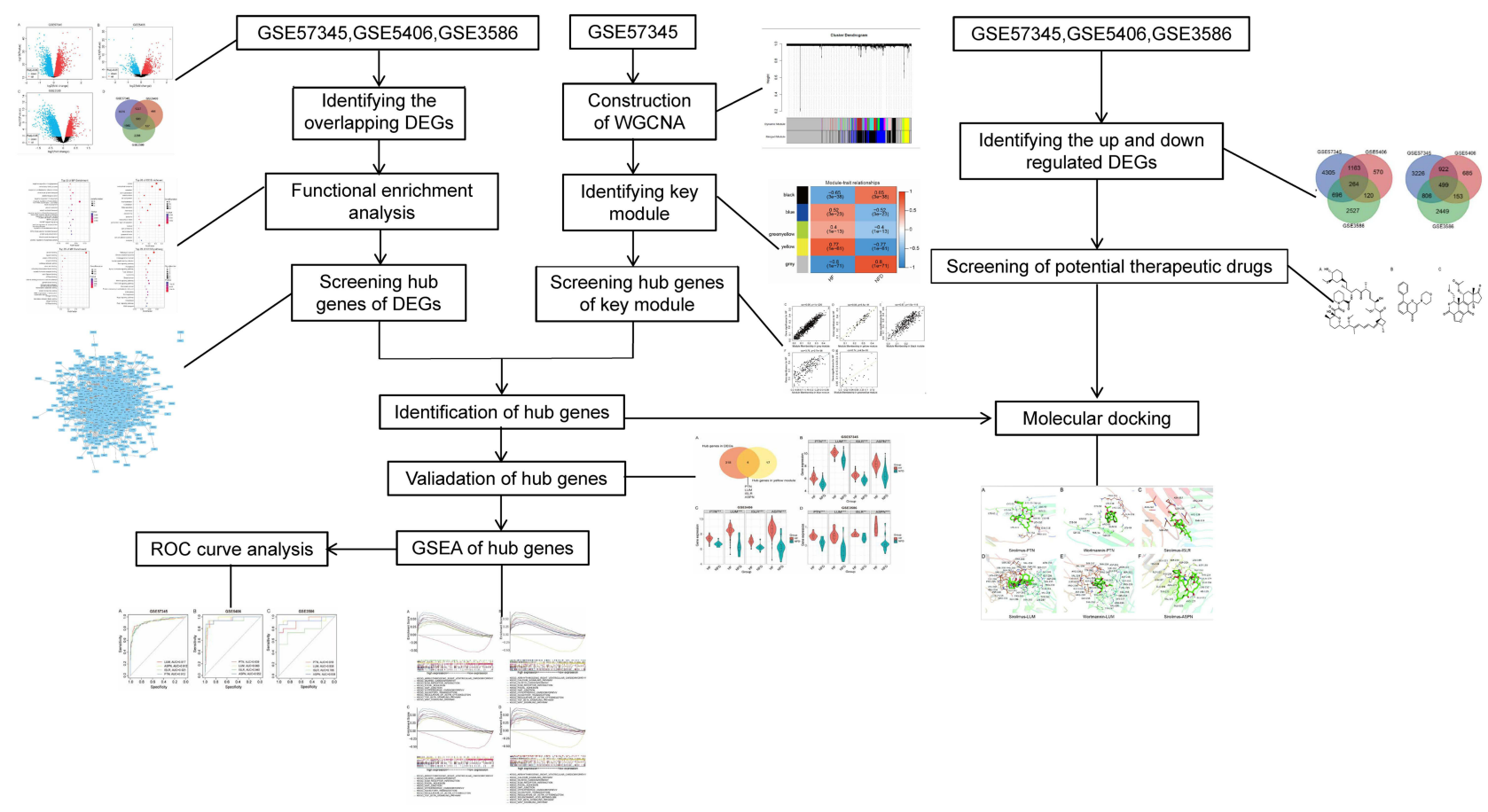

Figure I Flowchart of study outline. The flowchart constructed by Draw io online tool (http://www.draw.io/index.html). 


\section{Functional Enrichment Analysis of the Overlapping DEGs}

The overlapping DEGs were uploaded to the Database for Annotation, Visualization, and Integrated Discovery (DAVID, https://david.ncifcrf.gov/) and Metascape (http://metascape.org/) databases for Gene Ontology (GO) function and Kyoto Encyclopedia of Genes and Genomes (KEGG) pathway enrichment analyses respectively. $\mathrm{P}<0.05$ was considered statistically significant.

\section{PPI Network Analysis}

The protein-protein interaction (PPI) network was constructed using the STRING database, and visualized with the Cytoscape software (3.7.2). DEGs with connectivity $\geq$ 5 in the PPI network were considered to be the hub genes.

\section{WGCNA}

The R package WGCNA was used to construct the weight co-expression network of the GSE57345 dataset. The GSE57345 dataset was used in the WGCNA analysis because it contained the largest sample size. The weighing coefficient $\beta$ was first calculated based on $\mathrm{R}^{2}>0.9$ of the scale-free real biological network. After determining the adjacency function parameter $\beta$, a hierarchical clustering tree of different gene modules was constructed. The Pearson correlation coefficient was then used to transform the correlation matrix into an adjacency matrix and subsequently to a topological overlap matrix (TOM).

\section{Identification of Key Modules and Hub Genes}

The correlation among the genes in the aforementioned modules was analyzed and a heatmap was constructed. The module most closely related to the HF state was considered the key module of HF. To verify specific modulus-character associations, the correlation between GS and $\mathrm{MM}$ was investigated. Genes with $|\mathrm{MM}|>0.8$ were subsequently screened out as hub genes in the key module. The intersecting hub genes between the key module and the DEGs were finally defined as the hub genes of HF.

\section{Validation of Hub Genes}

We used the GSE57345, GSE5406, and GSE3586 datasets to confirm the identity of the hub genes that may be associated with HF. Then, we used the $t$-test to determine the significance of the correlation between the expression of hub genes and HF. Receiver operating characteristic
(ROC) curves were drawn for the core genes in the three datasets, and the area under the curve (AUC) was calculated. Specificity, sensitivity, and net reclassification index (NRI) were calculated to evaluate the value of the genetic diagnosis.

\section{Functional Analysis of Hub Genes by GSEA}

GSEA was performed to clarify the biological functions of the hub genes using the KEGG gene set (c2.cp. kegg. v7.2.) as the default and $p<0.05$, as the threshold. The HF samples of the GSE57345 data set were divided into high and low expression groups of each hub gene. The enrichment graph was plotted using the clusterProfiler package of the $\mathrm{R}$ language and GSEA function.

\section{Screening of Potential Therapeutic Drugs}

The CMap database includes 1309 compounds and expression data of $>7000$ human genes. The DEGs intersecting GSE57345, GSE5406, and GSE3585 datasets were uploaded to CMap. The negatively correlated small molecules were screened out using $\mathrm{p}<0.0001$ and mean $<0.4$ as the criteria. ChemBioDrawUltta 17.0 software (http:// www.chemdraw.com.cn) was used to draw the 3D structural formulas of potential therapeutic drugs and save them in mol2 format as small molecule compounds. The 3D crystal structures of the core targets were downloaded from the UniProt database (https://www.uniprot.org/). The Surflex-Dock module of SYBYL2.1 software was used for molecular docking, with total score $>4$ as the threshold for binding ability. The docking results were visualized using the Pymol software.

\section{Results Identification and Analysis of DEGs}

A total of 583 DEGs were identified between the HF and NFD samples across three GEO datasets (Figure 2A-D). The most significantly enriched GO terms pertaining to biological process (BP) were negative regulation of angiogenesis $(p=1.55 \mathrm{E}-04)$, response to endoplasmic reticulum stress $(p=6.63 \mathrm{E}-04)$, heart development $(p=0.002463)$, regulation of ventricular cardiac muscle cell action potential $(\mathrm{p}=0.004523)$, MAPK cascade $(\mathrm{p}=0.005025)$, and blood vessel development $(\mathrm{p}=0.007107)$ (Figure $3 \mathrm{~A}$ and Table S1). The cellular components (CC) terms including extracellular exosome ( $p=2.72 \mathrm{E}-08)$, cytoplasm ( $p$ $=9.57 \mathrm{E}-06)$, actin cytoskeleton p (p =4.94E-05), 

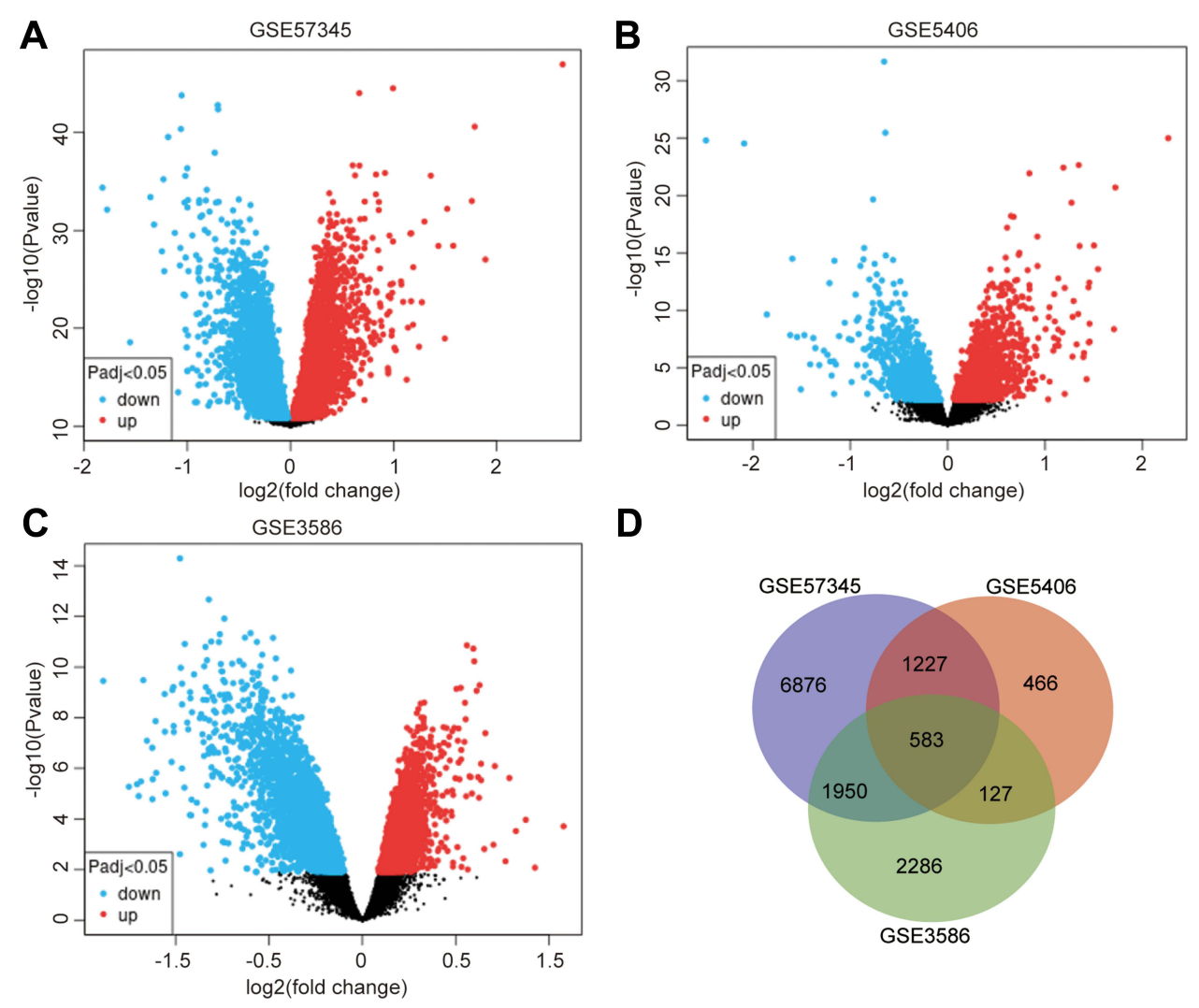

D

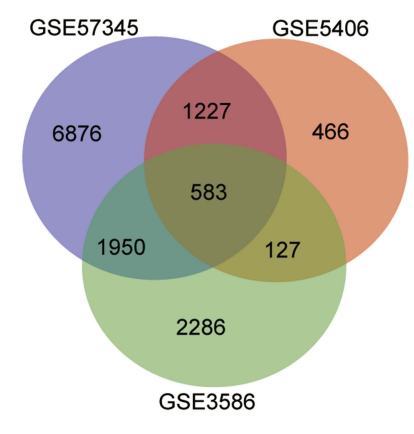

Figure 2 The DEGs between HF and NFD. The volcano plots of DEGs in (A) GSE57345, (B) GSE5406 and (C) GSE3586. (D) Venn diagrams of DEGs in three data sets.

mitochondrion ( $\mathrm{p}=8.84 \mathrm{E}-05)$, nucleoplasm $(\mathrm{p}=1.03 \mathrm{E}-04)$, Golgi apparatus $(\mathrm{p}=1.21 \mathrm{E}-04)$ and lysosome $(\mathrm{p}=6.84 \mathrm{E}-$ 04) were significantly enriched (Figure 3B and Table S1). The top enriched molecular function (MF) terms were activating transcription factor binding $(\mathrm{p}=0.003852)$, actin filament binding ( $\mathrm{p}=0.006599)$, cadherin binding involved in cell-cell adhesion $(p=0.00701)$, transcription coactivator activity $(\mathrm{p}=0.018332)$ and collagen binding $(\mathrm{p}$ $=0.034108$ ) (Figure $3 \mathrm{C}$ and Table S1). In addition, KEGG analysis revealed that the Ras signaling pathway ( $p$ $=5.15548 \mathrm{E}-07)$, Focal adhesion ( $\mathrm{p}=1.11814 \mathrm{E}-05)$, Lysosome $(\mathrm{p}=2.43906 \mathrm{E}-05)$, MAPK signaling pathway $(\mathrm{p}=4.23641 \mathrm{E}-05)$, PI3K-Akt signaling $(\mathrm{p}=4.85396 \mathrm{E}-$ $05)$, Protein processing in endoplasmic reticulum $(\mathrm{p}=$ 5.21303E-05) and Hippo signaling pathway (p $=7.03525 \mathrm{E}-05$ ) were significantly enriched among the DEGs (Figure 3D and Table S1).

\section{Construction of WGCNA Network}

A total of 1589 genes were screened from the GSE57345 dataset for WGCNA ( $<<0.05, \mid \log 2$ FoldChange $\mid>0.5)$. Sample clustering showed no significant differences in the WGCNA (Figure 4A). At $\beta=4$, the scale-free network fitting index $\mathrm{R}^{2}$ was 0.9 , and the average connectivity approached 0 , indicating that this value could obtain a scale-free network that met all requirements. Thus, $\beta=$ 4 was selected to construct a scale-free network (Figure 4B-C). A dynamic shearing algorithm was used to cluster the genes and module divisions.

\section{Identification and Visualization of the Key Module}

Five gene co-expression modules were finally obtained by calculating the module feature vector of each and merging similar modules (Figure 5A). The genes were clustered in the black, blue, yellow and green-yellow modules, and those that could not be clustered into any module were specified to the gray module. The yellow module with 73 genes showed the strongest correlation with HF $(r=0.77$, $\mathrm{p}=1 \mathrm{e}-61$ ) (Figure 5B), as well as with the clinical phenotype as per GS and MM analyses (cor $=0.96, \mathrm{p}=5.5 \mathrm{e}-$ 41; Figure $5 \mathrm{C}-\mathrm{G}$ ). The genes distributed in the upper right corner were closely related to HF pathogenesis, and are likely the key disease genes. Twenty-one genes in the yellow module were confirmed as hub genes. 


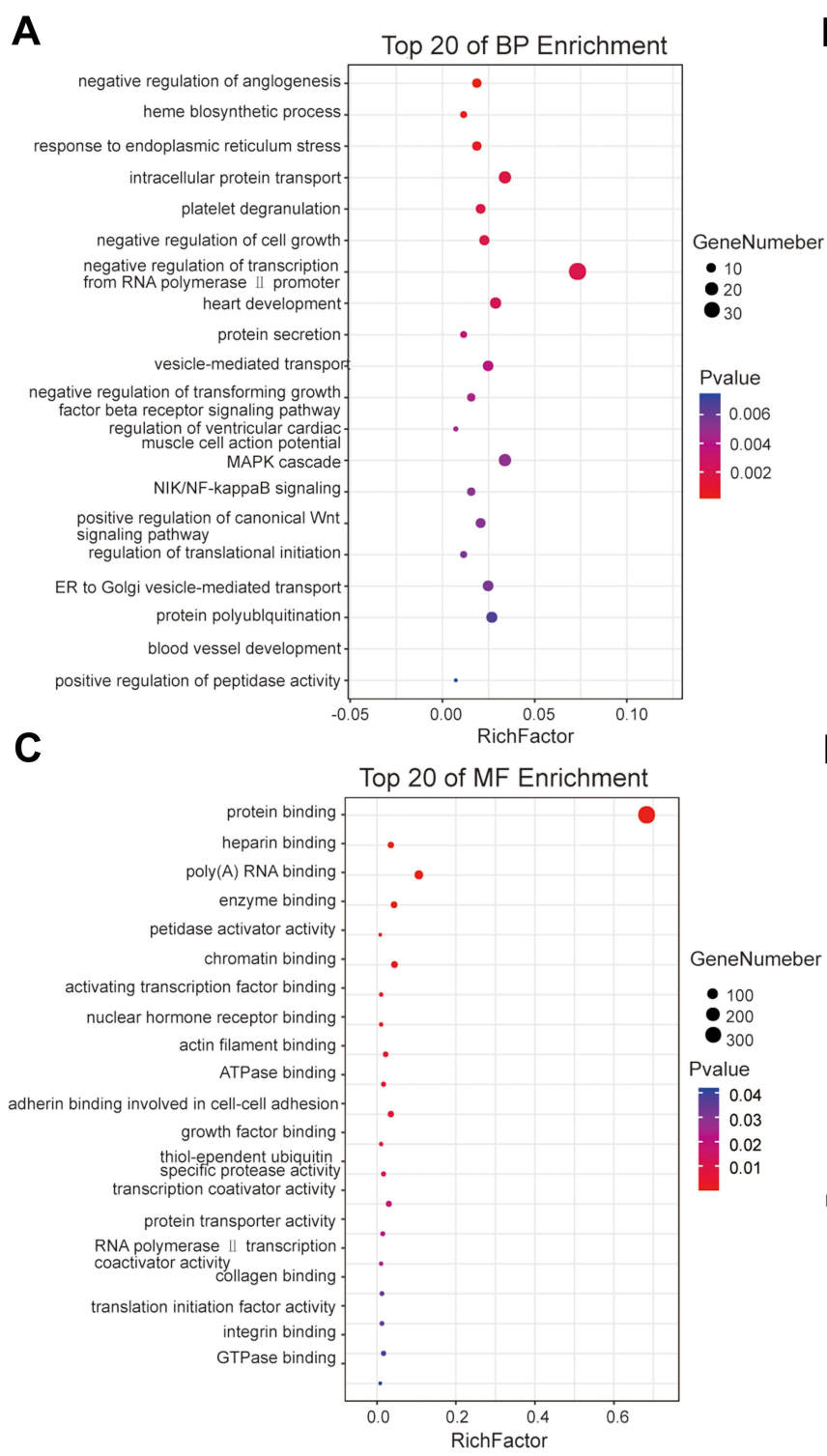

B
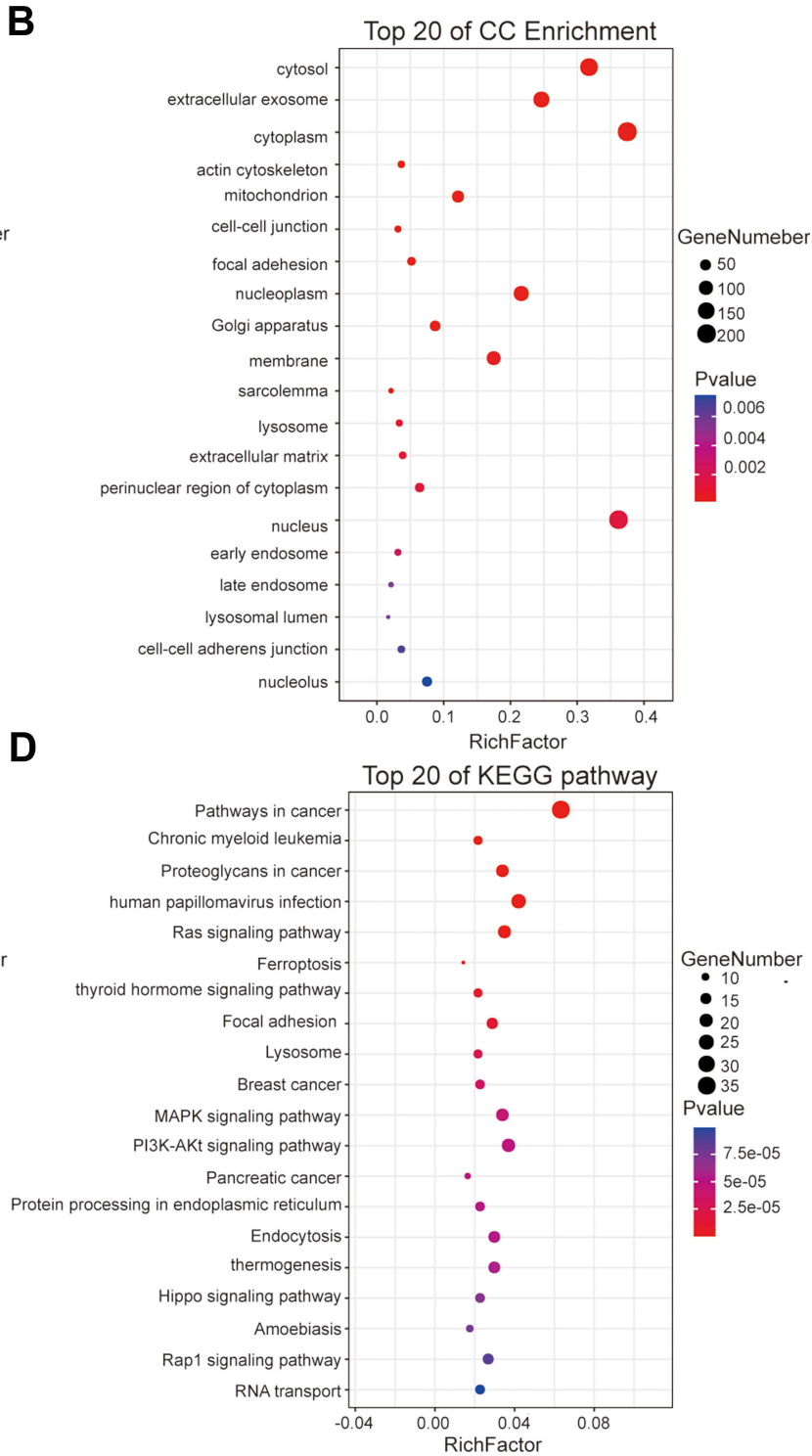

Figure $3 \mathrm{GO}$ and KEGG pathway enrichment analysis. Significantly enriched GO terms for (A) Biological processes, (B) Cellular component, (C) Molecular function. (D) KEGG pathway Molecular function $\mathrm{p}<0.05$ is considered statistically significant.

\section{Identification and Verification of Hub Genes}

From the 583 overlapping DEGs, 322 hub genes were selected for subsequent analysis (Figure 6A). The key intersecting genes between the 322 hub DEGs and 21 hub genes of the yellow module included PTN, LUM, ISLR and ASPN. The expression levels of these potentially key genes were analyzed in the HF and NFD samples of the GSE57345, GSE5406 and GSE3586 datasets. We further visualized their expression levels in the GSE57345 data set, and found that all four genes were overexpressed in HF samples compared to the NFD group $(\mathrm{p}<0.05$, Figure 6B). Afterwards, the genes were verified in GSE5406 and GSE3586, which verified higher expression levels in the HF group $(\mathrm{p}<0.05$, Figure $6 \mathrm{C}$ and $\mathrm{D})$.

\section{ROC Curve Analysis of Key Genes}

The potential diagnostic value of PTN, LUM, ISLR and ASPN was ascertained by plotting the ROC curve based on the expression data in GSE57345, GSE5406 and GSE3586. As shown in Figure $7 \mathrm{~A}-\mathrm{C}$, the AUC of all genes in all datasets exceeded 0.9 , except for 0.785 calculated for ISLR in GSE3586. We uploaded AUC and Standard Error data into MedCalc software, and applied 


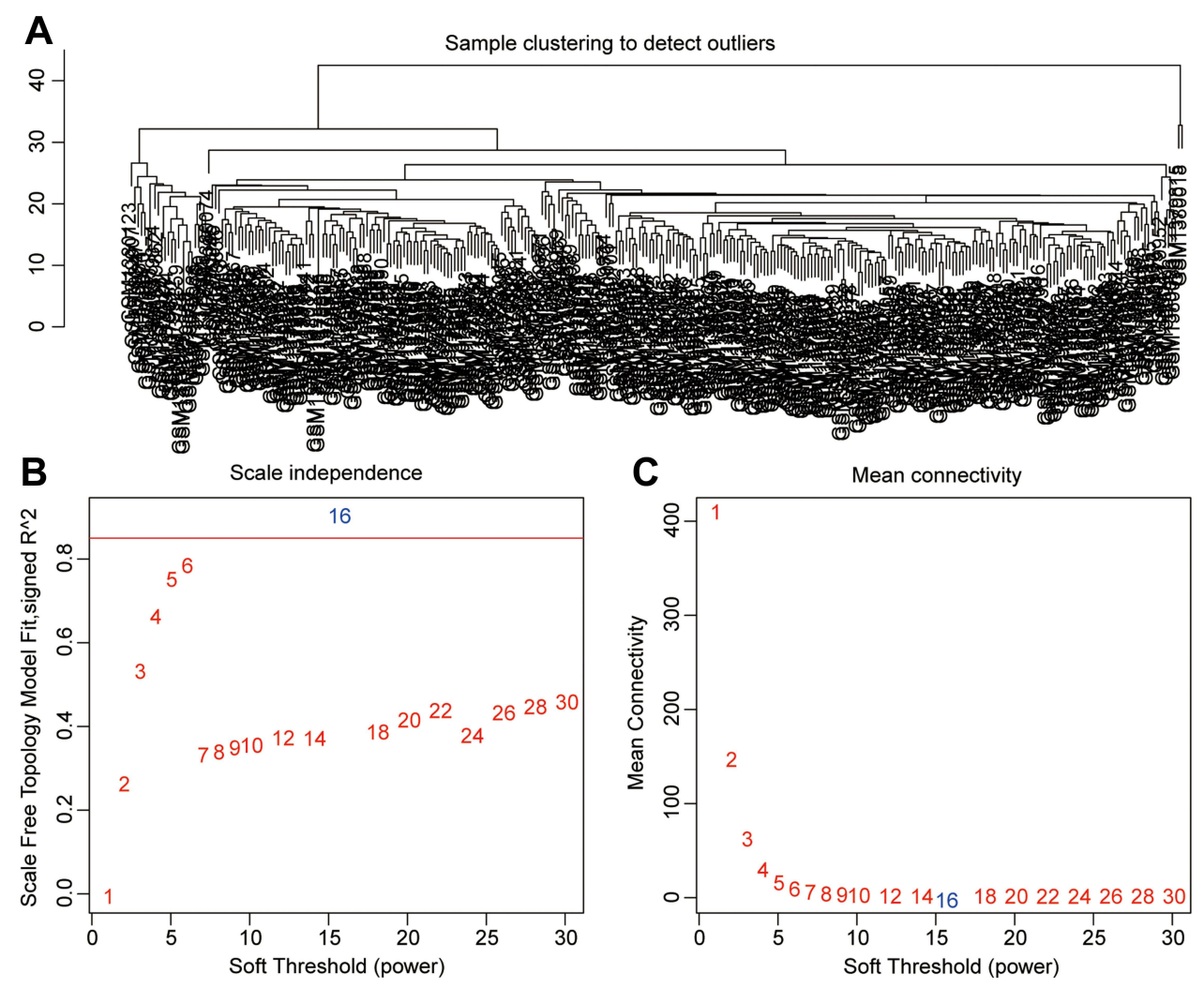

Figure 4 Determination of soft-threshold power $\beta$. (A) Clustering dendrogram of 319 samples. (B) Scale-free topology fit index as a function of the soft-threshold power. The red line indicates that $R^{2}$ is equal to 0.9 . (C) Mean connectivity as a function of the soft-threshold power.

the $\mathrm{Z}$ test to compare the expression of PTN, LUM, ISLR and APSN between the datasets. The results showed that there was no statistical difference $(p>0.05)$. We used the NRI to analyze differences in the expression levels of the four predicted HF hub genes in the GSE57345, GSE5406 and GSE3586 datasets. The PTN and ISLR of the GSE5406 dataset showed significant differences in predicting HF (NRI [95\% CI]: 0.3228 [0.0361-0.6095], p: 0.027); the prediction effects of the PTN and ISLR genes were significantly different, NRI [95\% CI]: 0.3905 [0.0073-0.7736], p: 0.045). The prediction effects of ISLR and LUM gene in the GSE5406 dataset were significantly different (NRI [95\% $\mathrm{CI}$ : -0.5077 [-0.9361-0.0793], p: 0.020). Subsequently, ROC analysis was performed to determine the diagnostic value of the four key genes, and the results suggested that these four hub genes can diagnose HF with high sensitivity and specificity (Table 1).

\section{GSEA of Hub Genes}

GSEA of PTN, LUM, ISLR and ASPN revealed direct involvement in the pathogenesis of HF. As shown in Figure $8 \mathrm{~A}-\mathrm{D}$, all genes were enriched in arrhythmogenic right ventricular cardiomyopathy (ARVC), dilated cardiomyopathy, ecm receptor interaction, focal adhesion, gap junction, hypertrophic cardiomyopathy (HCM), regulation of actin cytoskeleton and TGF- $\beta$ signaling pathway. In addition, PTN, LUM and ASPN were enriched in the WNT signal pathway, LUM in the calcium signaling pathway, and ASPN is likely involved in seleno-amino acid metabolism.

\section{Identification of Potential Therapeutic Drugs}

There were 264 upregulated and 499 down-regulated genes intersecting across the three datasets. The genes were uploaded to the cMap database to filter out negatively related small molecule compounds ( $\mathrm{p}<0.0001$ and mean $<0.4$ ). Sirolimus, LY-294002, and wortmannin were identified as potential drugs of HF (Figure 9A-C). Molecular docking showed that sirolimus had good affinity for PTN, ISLR, LUM, and ASPN, and wortmannin for PTN and LUM (Figure 9D). The molecular docking diagrams of potential compounds and hub targets are shown in Figure 9E-J. 
A

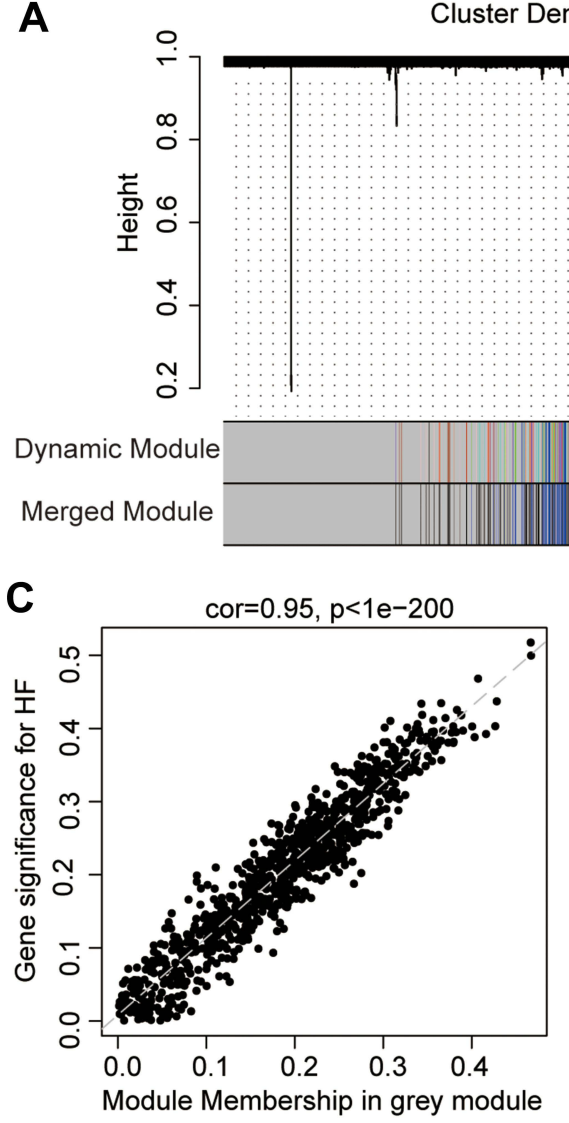

F $\quad c o r=0.75, p=2.7 e-38$

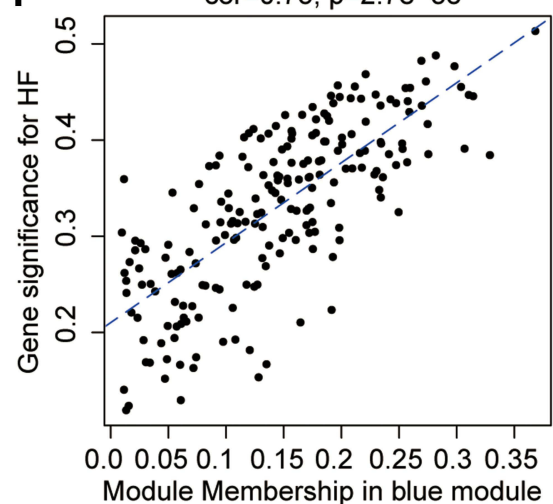

B

B

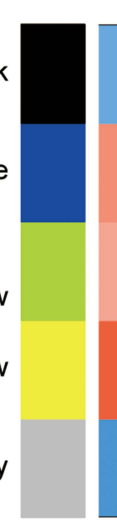

Module-trait relationships

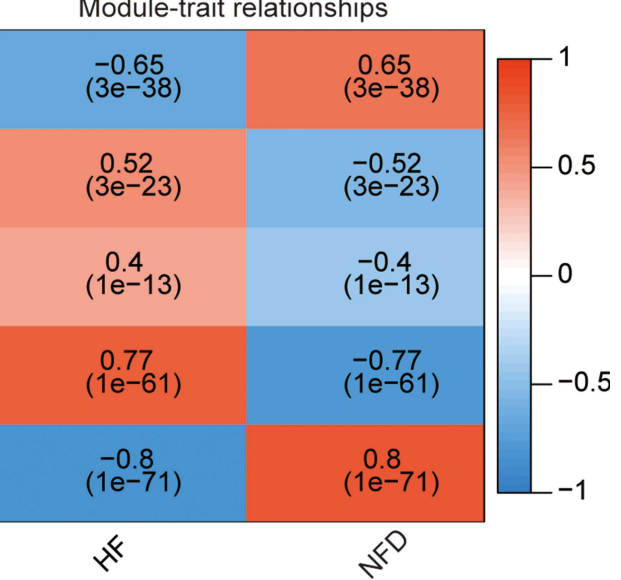

D
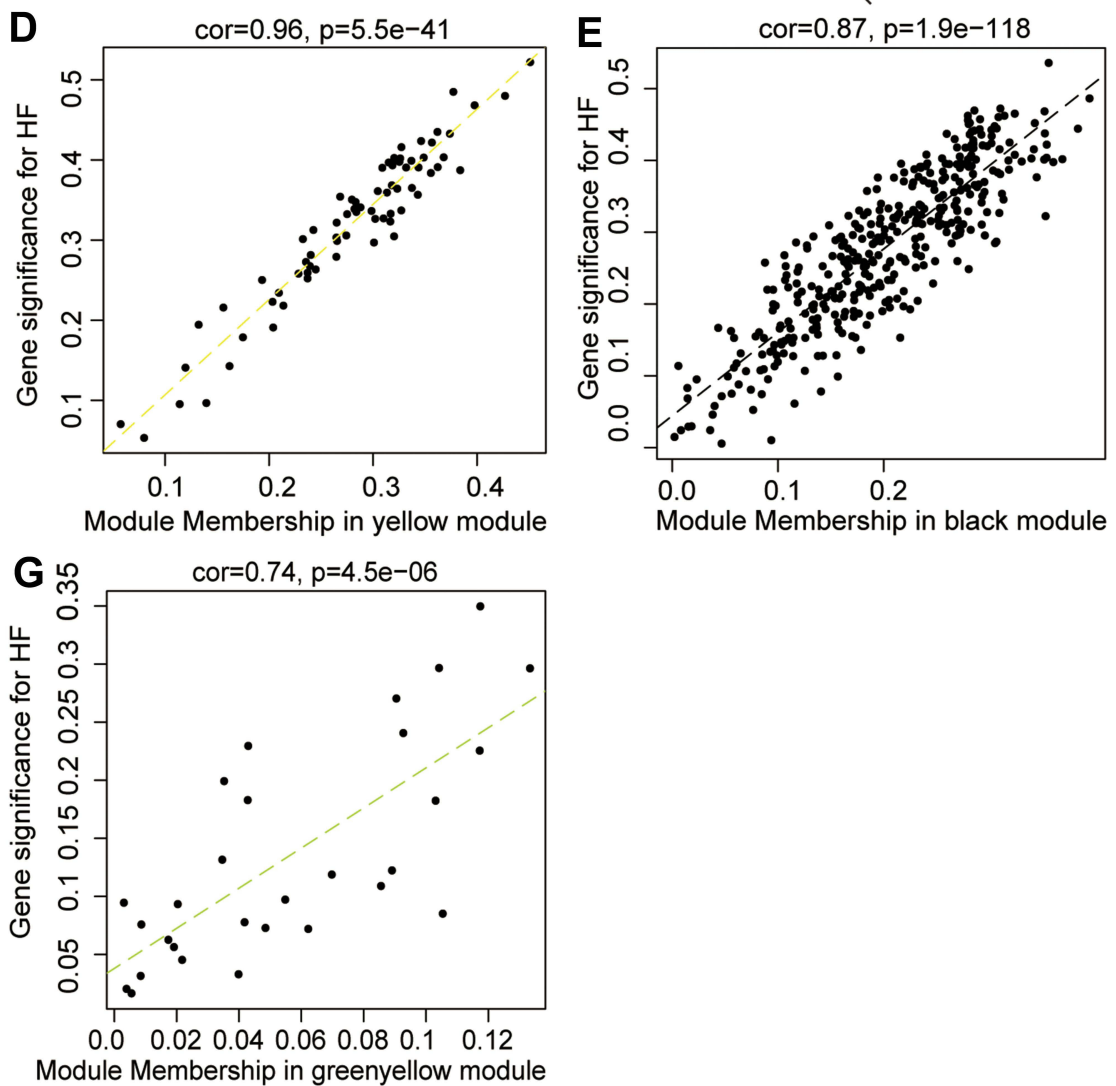

Figure 5 Identification of key HF gene modules. (A) Clustering dendrograms of genes and module detecting. (B) Heat map of the correlation between HF modules. (C-G) Correlation of GS and MM in the HF-related module. $\mathrm{p}<0.05$ is considered statistically significant.

\section{Discussion}

In this study, we combined WGCNA and DEGs to screen for genes associated with $\mathrm{HF}$ and found that the expression levels of the PTN, ISLR, LUM, and ASPN genes were all upregulated in HF. Further analysis using the ROC curve showed that these four genes may be potential biomarkers of HF. At present, PTN and ISLR have not been reported to be associated with HF, but there is evidence that they may be potentially associated with HF. Single-gene GSEA showed that the hub genes of HF are related to arrhythmic right ventricular cardiomyopathy, dilated cardiomyopathy and hypertrophic cardiomyopathy, which is consistent with reports demonstrating that these cardiovascular disorders precede the final HF stage. ${ }^{13-15}$ Single-gene GSEA showed that the hub genes of HF are related to arrhythmic right. 
A

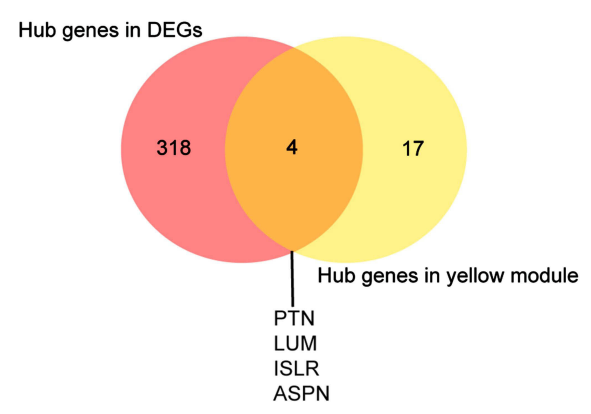

C

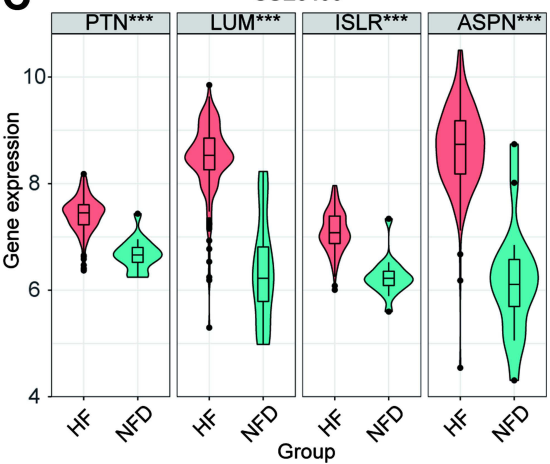

B

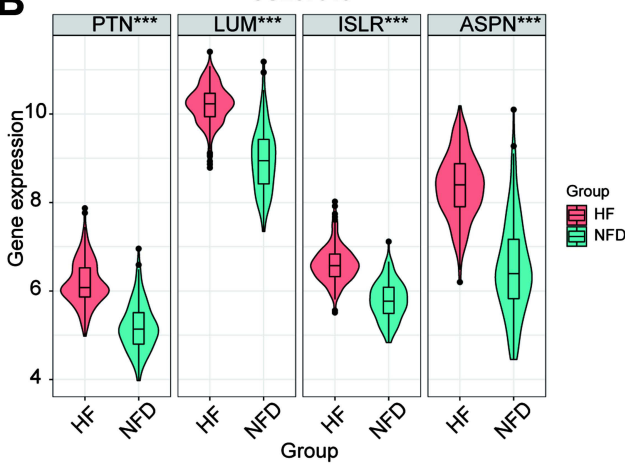

D

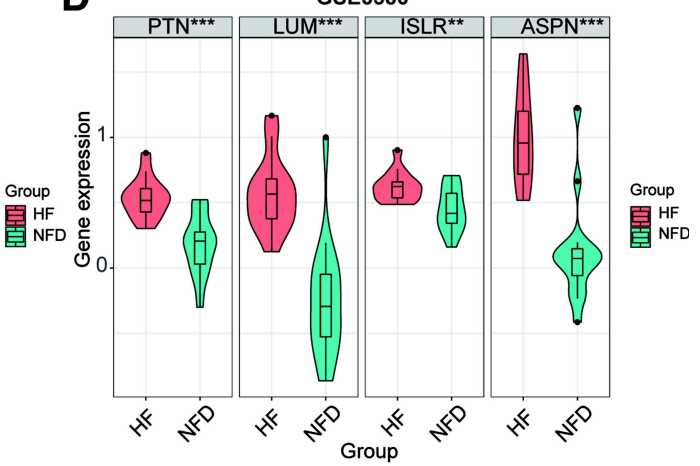

Figure 6 Analysis of key genes. (A) The Venn diagram of hub genes in the yellow module and hub genes in DEGs. Expression of PTN, LUM, ISLR and ASPN in the (B) GSE57345, (C) GSE5406 and (D) GSE3586 datasets. **p $<0.01$ and $* * * \mathrm{p}<0.001$ are considered statistically significant.
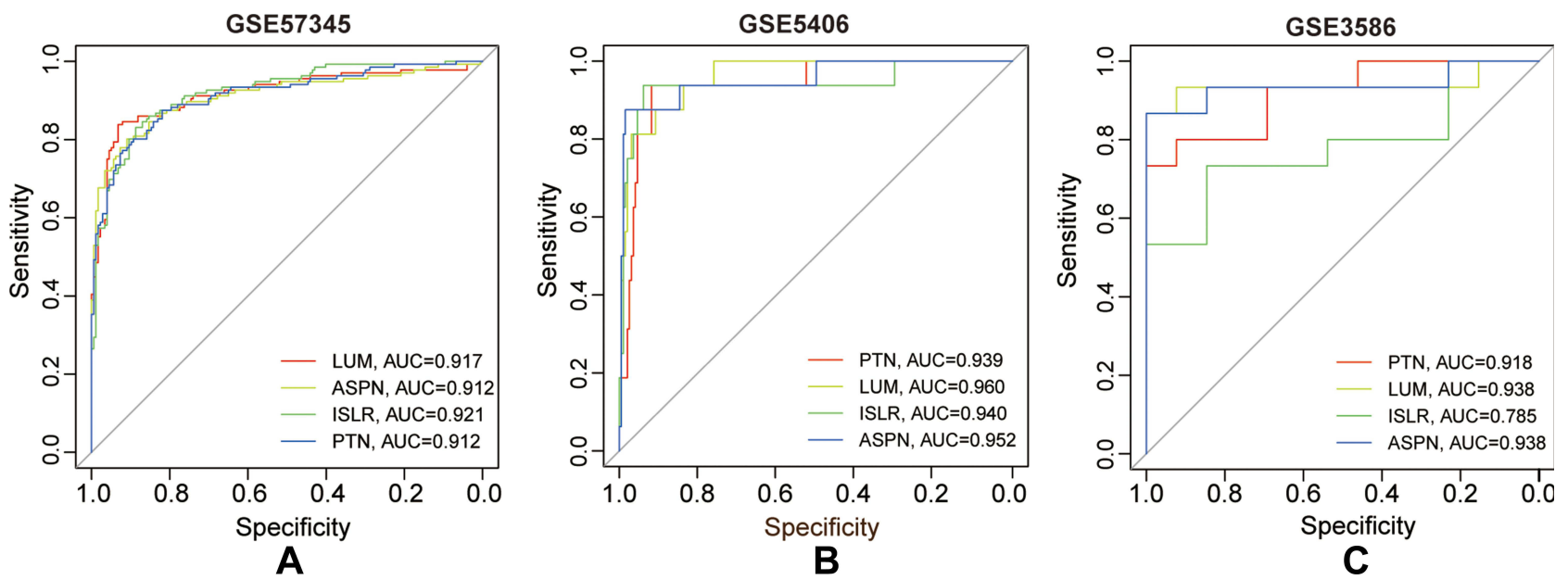

Figure 7 The ROC curve of hub genes (A) GSE57345. (B) GSE5406. (C) GSE3586. The x-axis shows specificity, and the $y$-axis shows sensitivity. Abbreviations: ROC, receiver operating characteristic; AUC: area under the ROC curve.

GO analysis of the DEGs showed that endoplasmic reticulum stress (ERS) is closely related to HF pathogenesis, which is consistent with previous reports. ${ }^{16-18}$ The risk factors of HF can induce ERS in myocardial cells, which culminates in apoptosis and cardiovascular dysfunction. In addition, the DEGs were enriched in ferroptosis, MAPK signaling pathway, PI3K-Akt signaling pathway, and the Hippo signaling pathway, all of which are involved in HF. Liu et al ${ }^{19}$ detected a high level of ferroptosis in the cardiomyocytes of a rat model of pressure overload-induced HF. Exogenous expression of ferritin FTH1 and GPX4 and reduction in ROS levels through NOX4 knockdown inhibited ferroptosis in cardiomyocytes and improved cardiac function. Fang and Koleini et $\mathrm{al}^{20,21}$ found that doxorubicin induced the accumulation of oxidized phospholipids in 
Table I ROC Curve Analysis of Hub Genes

\begin{tabular}{|l|c|c|c|c|c|c|c|}
\hline Data set & Gene & Specificity (\%) & Sensitivity (\%) & Youden Index & AUC & Standard Error & 95\% CI \\
\hline GSE57345 & PTN & 87.5 & 81.9 & 0.6942 & 0.912 & 0.0171 & $0.875-0.941$ \\
& LUM & 83.8 & 93.2 & 0.7704 & 0.917 & 0.0177 & $0.881-0.945$ \\
& ISLR & 83.1 & 88.7 & 0.7179 & 0.921 & 0.0155 & $0.886-0.949$ \\
& ASPN & 80.1 & 91.0 & 0.7111 & 0.912 & 0.0182 & $0.875-0.941$ \\
\hline \multirow{2}{*}{ GSE5406 } & PTN & 91.8 & 93.7 & 0.8550 & 0.939 & 0.0303 & $0.898-0.967$ \\
& LUM & 90.7 & 87.5 & 0.7822 & 0.960 & 0.0187 & $0.924-0.982$ \\
& ISLR & 93.8 & 93.7 & 0.8756 & 0.940 & 0.0438 & $0.899-0.968$ \\
& ASPN & 98.5 & 87.5 & 0.8595 & 0.952 & 0.0324 & $0.914-0.977$ \\
\hline \multirow{2}{*}{ GSE3586 } & PTN & 73.3 & 100 & 0.7333 & 0.918 & 0.0508 & $0.751-0.988$ \\
& LUM & 86.7 & 100 & 0.8667 & 0.938 & 0.057 & $0.779-0.994$ \\
& ISLR & 73.3 & 84.6 & 0.5795 & 0.785 & 0.0909 & $0.589-0.916$ \\
& ASPN & 86.7 & 100 & 0.8667 & 0.938 & 0.0529 & $0.779-0.994$ \\
\hline
\end{tabular}

Abbreviations: AUC, Area under the ROC curve; $95 \% \mathrm{Cl}$, $95 \%$ Confidence interval.

undifferentiated cardiomyocytes and up-regulated heme oxygenase1 (HMOX1), resulting in heme degradation, free iron overload, and ferroptosis, which eventually leads to HF. The loss of myeloid differentiation protein 1 (MD1) activates ROS and exacerbates autophagy induced by the MAPK signaling pathway. Therefore, the MD1-ROS-MAPK axis is a novel therapeutic target for HF that can preserve the ejection fraction. ${ }^{22}$ Mao Liu et $\mathrm{al}^{23}$ showed that paeoniflorin reduced myocardial fibrosis and improved cardiac function in rats with chronic HF by regulating the p38/MAPK signaling pathway. Apelin-13 can slow down oxidative stress by inhibiting the PI3K/Akt signaling pathway in the rat $\mathrm{HF}$ model and ameliorate angiotensin II-induced cardiac insufficiency, impaired cardiac hemodynamics, and fibroblast fibrosis. ${ }^{24}$ Hou and Li et al ${ }^{25,26}$ showed that YAP/ TAZ can initiate the transcription of connective tissue growth factor by interacting with the TEAD domain family, increase the expression of extracellular matrix genes, promote cardiac remodeling and fibrosis, and thus delay the progression of HF. Leach et $\mathrm{al}^{27}$ found that knocking out the SALV gene increased the number of left ventricular myocardial cells in mice with myocardial infarction, which reduced ventricular fibrosis and increased the number of new capillaries around the injured myocardium, indicating that the Hippo signaling pathway can enhance heart function.

PTN is a highly conserved proto-oncogene closely related to tumor angiogenesis and metastasis. ${ }^{28}$ It is highly expressed in various malignant tumors, such as breast cancer, prostate cancer and rectal cancer, ${ }^{29,30}$ and promotes the proliferation, mitosis, differentiation, and migration of vascular endothelial cells. ${ }^{31}$ Overexpression of PTN gene can promote bone formation, whereas PTN gene knockout mice have dysfunctional bone growth and remodeling. ${ }^{32}$ LUM is a member of the SLRP family of leucine-rich proteins that are secreted by the extracellular matrix. It is widely distributed in various tissues, and shows aberrant expression levels in pancreatic cancer, colorectal cancer, breast cancer and cervical cancer. ${ }^{33}$ LUM has both oncogenic and tumor-suppressive functions depending on the cancer type. For instance, LUM facilitated the metastasis of colon cancer cells by reconstructing the actin cytoskeleton, but inhibited the adhesion of osteosarcoma cells via the TGF- $\beta 2$ signaling pathway. ${ }^{34,35}$ ISLR is a conserved immune-related protein that is mainly expressed in stromal cells. ${ }^{36} \mathrm{Xu}$ et $\mathrm{al}^{37}$ showed that ISLR can inhibit Hippo signal transduction during intestinal regeneration and tumorigenesis and activate YAP factor in epithelial cells. Knocking out ISLR in mouse stromal cells significantly affected intestinal regeneration and inhibited colorectal tumorigenesis. Zhang and Hara et $\mathrm{al}^{38,39}$ further showed that the ISLR can promote muscle regeneration and improve myocardial tissue repair. However, little is known regarding the correlation between ISLR and HF. ASPN is an extracellular matrix protein and a member of the leucine-rich small proteoglycan family. ${ }^{40}$ Sasaki et $\mathrm{al}^{41}$ showed that ASPN protected gastric tumor cells against oxidative stress by up-regulating HIF $1 \alpha$ and reducing the levels of mitochondrial ROS. It also increased the expression of CD44 to accelerate the migration and infiltration of gastric cancer cells. However, other 


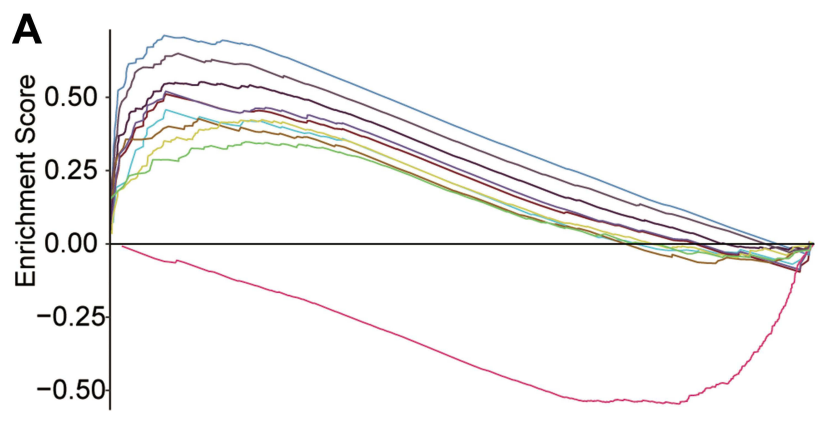

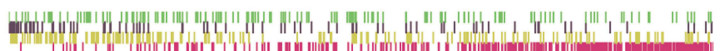

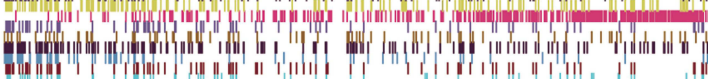
high expression<------------->low expression

- KEGG_ARRHYTHMOGENIC_RIGHT_VENTRICULAR_CARDIOMYOPATHY - KEGG_DILATED_CARDIOMYOPATHY

- KEGG_ECM_RECEPTOR_INTERACTION

- KEGG_FOCAL_ADHESION

- KEGG_GAP JŪNCTION

- KEGG_HYPERTROPHIC_CARDIOMYOPATHY

- KEGG_OLFACTORY TRANSDUCTION

- KEGG_REGULATION_OF_ACTIN_CYTOSKELETON

- KEGG_TGF_BETA_SIGNÄLING_PATHWAY

- KEGG_WNT_SIGNALING_PATHWAY

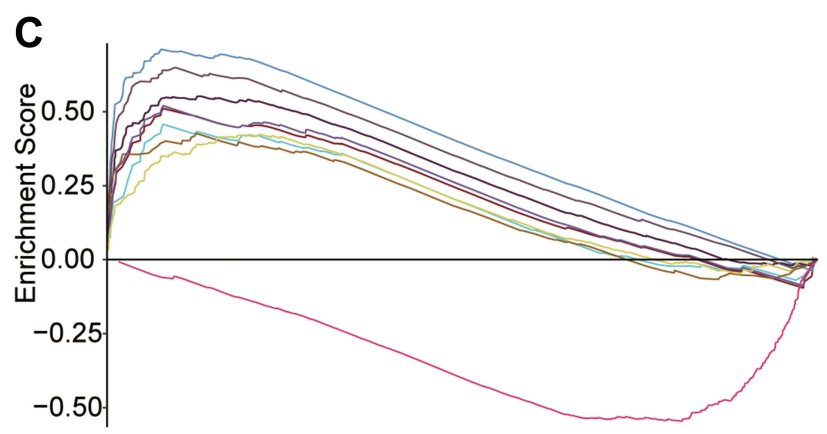

|

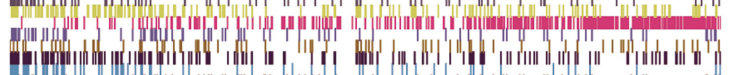

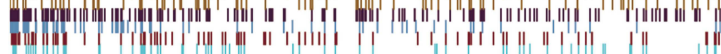

high expression<----------->low expression

— KEGG_ARRHYTHMOGENIC_RIGHT_VENTRICULAR_CARDIOMYOPATHY

- KEGG_DILATED_CARDIOMYOPATHY

- KEGG_ECM_RECEEPTOR_INTERACTION

- KEGG_FOCĀL_ADHESION

- KEGG_GAP JŪNCTION

- KEGG_HYPËRTROPHIC CARDIOMYOPATHY

- KEGG_OLFACTORY_TRANSDUCTION

- KEGG REGULATION OF ACTIN CYTOSKELETON

- KEGG_TGF_BETA_SIGNALING_PATHWAY

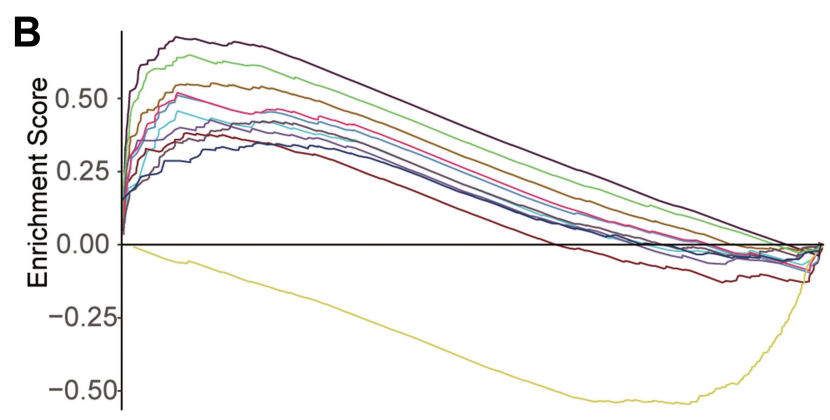

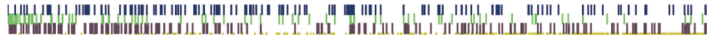

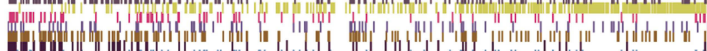

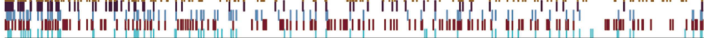
high expression<------------>low expression

- KEGG_ARRHYTHMOGENIC_RIGHT_VENTRICULAR_CARDIOMYOPATHY - KEGG_CALCIUM_SIGNALING_PATHWAY

- KEGG_DILATED_CARDIOMYOPATHY

- KEGG_ECM_RECEPTOR_INTERACTION

- KEGG_FOCĀL_ADHESION

- KEGG_GAP_JUNNCTION

- KEGG_HYPERTROPHIC_CARDIOMYOPATHY

- KEGG_OLFACTORY_TRANNSDUCTION

- KEGG_REGULATION OF ACTIN CYTOSKELETON

- KEGG TGF BETA SIGNĀLING_PATHWAY

- KEGG_WNT'SIGNALING PATHWAY

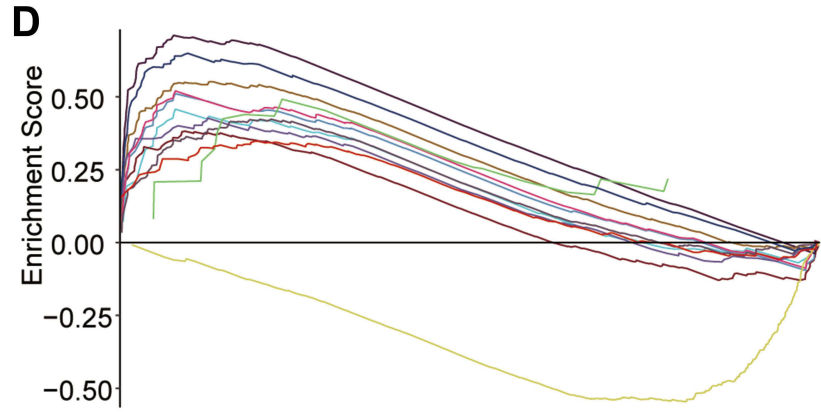

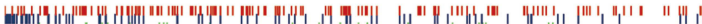
-

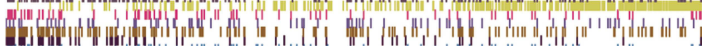

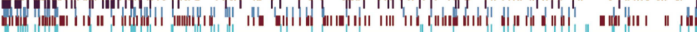
high expression<------------>low expression

- KEGG_ARRHYTHMOGENIC_RIGHT_VENTRICULAR_CARDIOMYOPATHY - KEGG CALCIUM SIGNALING PATHWAY

- KEGG_DILATED CARDIOMYOPATHY

- KEGG ECM RECEPTOR INTERACTION

- KEGG FOCÁL ADHESION

- KEGG GAP JŪNCTION

- KEGG_HYPERTROPHIC_CARDIOMYOPATHY

- KEGG_OLFACTORY_TRĀNSDUCTION

- KEGG_REGULATION_OF_ACTIN_CYTOSKELETON

- KEGG_SELENOAMINO_ACID_METTABOLISM

- KEGG_TGF_BETA_SIGNALING PATHWAY

- KEGG WNT SIGNALING PATHWAY

Figure 8 Results of GSEA. (A) PTN. (B) LUM. (C) ISLR. (D) ASPN.

reports indicate an anti-tumorigenic role of ASPN in breast cancer. $^{42,43}$ Studies also show that ASPN is up-regulated during aortic stenosis or coronary artery ligation in ischemic cardiomyopathy patients and animal models. ${ }^{44}$ ASPN may also increase the apoptosis and fibrosis of H9C2 cardiomyocytes. ${ }^{45}$ However, the exact role of ASPN in HF pathogenesis needs further investigation.
Lu A et a ${ }^{46}$ found that Wnt3a binds to FZD and LRP5/ 6 receptors, thereby activating the classic Wnt-Dvl- $\beta$ catenin signaling pathway and promoting myocardial hypertrophy. Wnt signaling can inhibit $\mathrm{Na}^{+}$channels by directly or indirectly inhibiting the expression of Scn5a. Thus, blocking these intracellular cascades is a rational therapeutic strategy against $\mathrm{HF}^{46} \mathrm{He}$ et $\mathrm{al}^{47}$ found that 


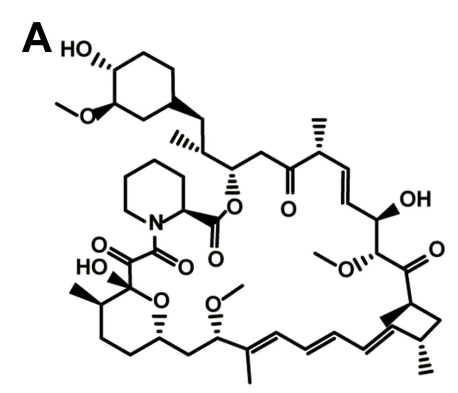

$\mathbf{E}$

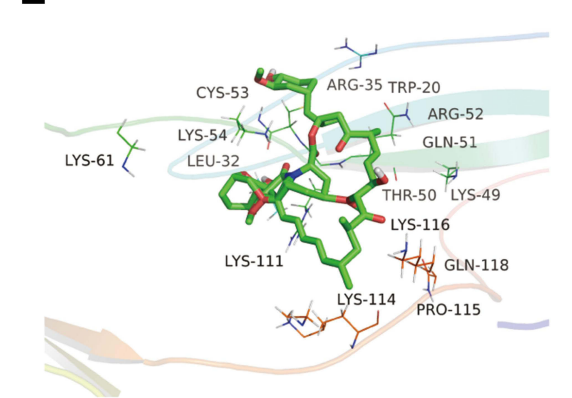

H

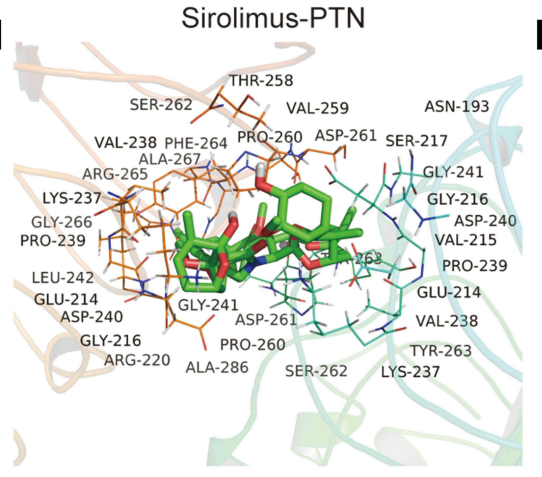

Sirolimus-LUM
B C

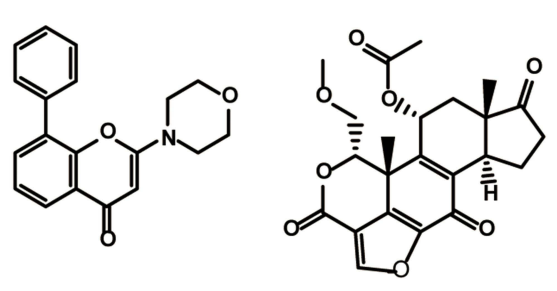

$\mathbf{F}$

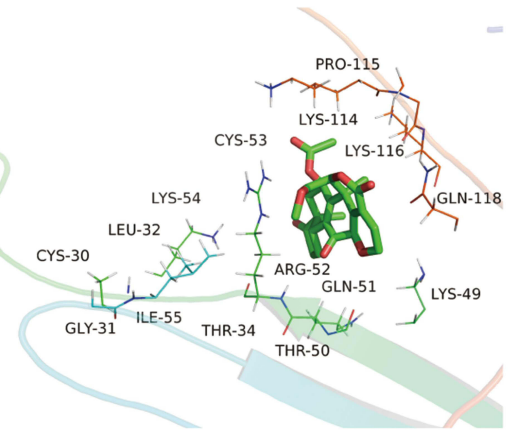

I
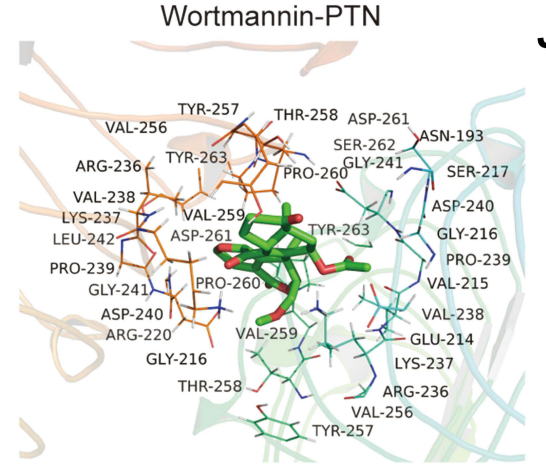

Wortmannin-LUM
D

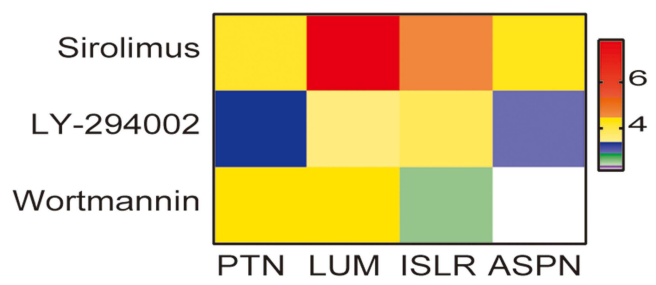

$-\mathbf{G}$

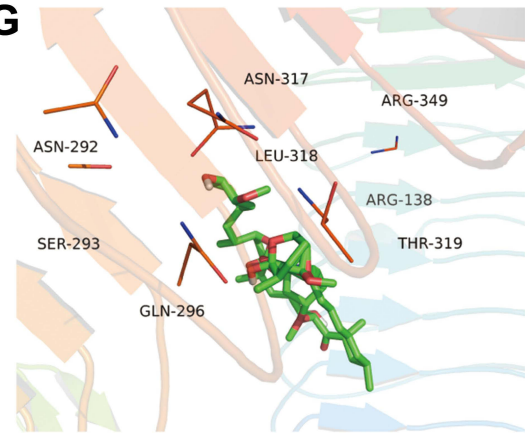

$\mathbf{J}$

Sirolimus-ISLR

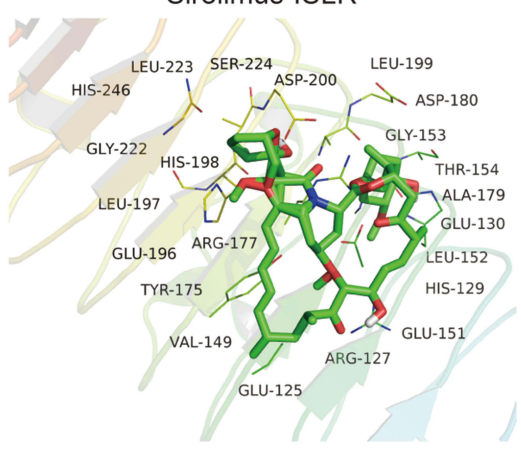

Sirolimus-ASPN

Figure 9 The potential therapeutic drugs of HF. (A-C) The 2D structure of Sirolimus, LY-294002 and Wortmannin. (D) Heat map of the docking score between potential drugs and hub targets. The intensity of red color indicates binding ability. (E-J) Molecular docking diagram of certain core compounds and hub targets.

Wnt3a and Wnt5a ligand were up-regulated in a mouse model of cardiac hypertrophy, underscoring the role of the Wnt signaling pathway in HF pathogenesis. TGF- $\beta$ is an important factor regulating myocardial fibrosis, which gradually worsens during HF and alters cardiac function from the compensatory phase to the decompensated phase. ${ }^{48}$ Kakhi et $\mathrm{al}^{49}$ found that sirolimus, an mTOR inhibitor, reversed new HF after kidney transplantation in mammals. Gao et $\mathrm{al}^{50}$ also showed that rapamycin (sirolimus) can reduce cardiomyocyte apoptosis and promote autophagy by regulating mTOR and ERS, thus preventing myocardial damage caused by chronic HF. LY294002 and wortmannin are protein kinase inhibitors that block the PI3K signaling pathway. Melatonin alleviates cardiac hypertrophy by inhibiting the Akt/mTOR pathway and reducing Atg5dependent autophagy, which can be reversed by
LY294002. ${ }^{51}$ Studies show that apelin may reduce the myocardial damage caused by acute HF by regulating the APJ/Akt/ERS signaling pathway. However, wortmannin and LY294002 can reverse the cardioprotective effects of apelin. $^{52}$ The PI3K-Akt signaling pathway was also enriched among the HF-related DEGs, indicating a vital mechanistic role in its pathological progression. Molecular docking showed that sirolimus and wortmannin had a high affinity to the hub targets, LY-294002 bound weakly and may therefore have other targets. Nevertheless, all three drugs could be potentially effective for treating HF.

There are several limitations in this study. First, the data used in this study was obtained from the GEO database, which lacks clinical, in vivo, and in vitro experimental research certifications for pivotal genes and HF-associated genes. Second, the datasets used in this study were relatively 
small, and a larger sample size is needed to verify our results. However, our findings provide new insights into the underlying molecular mechanisms of $\mathrm{HF}$, along with potential diagnostic biomarkers and candidate therapeutic drugs, which will help provide new clues for HF research, diagnosis and treatment, and target selection.

\section{Conclusion}

PTN, LUM, ISLR, and ASPN are overexpressed in HF patients compared to NFD, and are mainly related to the TGF- $\beta$ and Wnt signaling pathways. Sirolimus, LY294002, and wortmannin are potential drug candidates for HF treatment. The in silico data will need to be verified by functional and clinical studies.

\section{Data Sharing Statement}

All datasets generated and analyzed during the current study were uploaded with the manuscript as additional files.

\section{Ethics Statement}

The ethics committee of the Qinghai University has waived the need for ethical approval for the reasons that the present study used public database, so it did not involve ethics.

\section{Funding}

This research was supported by the Technology Department project of Qinghai Science (No. 2020-ZJ -922).

\section{Disclosure}

The authors report no conflicts of interest in this work.

\section{References}

1. Bragazzi NL, Zhong W, Shu J, et al. Burden of heart failure and underlying causes in 195 countries and territories from 1990 to 2017. Eur J Prev Cardiol. 2021;28(15):1682-1690. doi:10.1093/eurjpc/zwaa147

2. Baman JR, Ahmad FS. Heart failure. JAMA. 2020;324(10):1015. doi:10.1001/jama.2020.13310

3. Brann A, Tran H, Greenberg B. Contemporary approach to treating heart failure. Trends Cardiovasc Med. 2020;30(8):507-518. doi:10. 1016/j.tcm.2019.11.011

4. Servant N, Roméjon J, Gestraud P, et al. Bioinformatics for precision medicine in oncology: principles and application to the SHIVA clinical trial. Front Genet. 2014;5:152. doi:10.3389/fgene.2014.00152

5. Yin X, Wang P, Yang T, et al. Identification of key modules and genes associated with breast cancer prognosis using WGCNA and ceRNA network analysis. Aging. 2020;13(2):2519-2538. doi:10.18632/aging.202285

6. Bai KH, He SY, Shu LL, et al. Identification of cancer stem cell characteristics in liver hepatocellular carcinoma by WGCNA analysis of transcriptome stemness index. Cancer Med. 2020;9(12):4290-4298. doi:10.1002/cam4.3047
7. Nangraj AS, Selvaraj G, Kaliamurthi S, et al. Integrated PPI- and WGCNA-retrieval of hub gene signatures shared between Barrett's esophagus and esophageal adenocarcinoma. Front Pharmacol. 2020;11:881. doi:10.3389/fphar.2020.00881

8. Zhang K, Qin X, Wen P, et al. Systematic analysis of molecular mechanisms of heart failure through the pathway and network-based approach. Life Sci. 2021;265:118830. doi:10.1016/j. Ifs. 2020.118830

9. Zhou J, Zhang W, Wei C, et al. Weighted correlation network bioinformatics uncovers a key molecular biosignature driving the left-sided heart failure. BMC Med Genomics. 2020;13(1):93. doi:10.1186/s12920-020-00750-9

10. Liu Y, Morley M, Brandimarto J, et al. RNA-seq identifies novel myocardial gene expression signatures of heart failure. Genomics. 2015;105(2):83-89. doi:10.1016/j.ygeno.2014.12.002

11. Hannenhalli S, Putt ME, Gilmore JM, et al. Transcriptional genomics associates FOX transcription factors with human heart failure. Circulation. 2006;114(12):1269-1276. doi:10.1161/ CIRCULATIONAHA.106.632430

12. Barth AS, Kuner R, Buness A, et al. Identification of a common gene expression signature in dilated cardiomyopathy across independent microarray studies. J Am Coll Cardiol. 2006;48(8):1610-1617. doi:10.1016/j.jacc.2006.07.026

13. Marian AJ, Braunwald E. Hypertrophic cardiomyopathy: genetics, pathogenesis, clinical manifestations, diagnosis, and therapy. Circ Res. 2017;121(7):749-770. doi:10.1161/CIRCRESAHA.117.311 059

14. Hänselmann A, Veltmann C, Bauersachs J, et al. Dilated cardiomyopathies and non-compaction cardiomyopathy. Herz. 2020;45 (3):212-220. doi:10.1007/s00059-020-04903-5

15. Zoppo F, Gagno G, Perazza L, et al. Electroanatomic voltage mapping and characterisation imaging for "right ventricle arrhythmic syndromes" beyond the arrhythmia definition: a comprehensive review. Int $J$ Cardiovasc Imaging. 2021;37(8):2347-2357. doi:10. 1007/s10554-021-02221-3

16. Goh KY, Qu J, Hong $\mathrm{H}$, et al. Impaired mitochondrial network excitability in failing Guinea-pig cardiomyocytes. Cardiovasc Res. 2016;109(1):79-89. doi:10.1093/cvr/cvv230

17. Bertero E, Maack C. Metabolic remodelling in heart failure. Nat Rev Cardiol. 2018;15(8):457-470. doi:10.1038/s41569-018-0044-6

18. Gutiérrez T, Parra V, Troncoso R, et al. Alteration in mitochondrial $\mathrm{Ca}(2+)$ uptake disrupts insulin signaling in hypertrophic cardiomyocytes. Cell Commun Signal. 2014;12:68. doi:10.1186/ s12964-014-0068-4

19. Liu B, Zhao C, Li H, et al. Puerarin protects against heart failure induced by pressure overload through mitigation of ferroptosis. Biochem Biophys Res Commun. 2018;497(1):233-240. doi:10.1016/ j.bbrc.2018.02.061

20. Fang X, Wang H, Han D, et al. Ferroptosis as a target for protection against cardiomyopathy. Proc Natl Acad Sci U S A. 2019;116 (7):2672-2680. doi:10.1073/pnas.1821022116

21. Koleini N, Nickel BE, Edel AL, et al. Oxidized phospholipids in Doxorubicin-induced cardiotoxicity. Chem Biol Interact. 2019;303:35-39. doi:10.1016/j.cbi.2019.01.032

22. Yang HJ, Kong B, Shuai W, et al. MD1 deletion exaggerates cardiomyocyte autophagy induced by heart failure with preserved ejection fraction through ROS/MAPK signalling pathway. J Cell Mol Med. 2020;24(16):9300-9312. doi:10.1111/jcmm.15579

23. Liu M, Feng J, Du Q, et al. Paeoniflorin attenuates myocardial fibrosis in Isoprenaline-induced chronic heart failure rats via inhibiting P38 MAPK pathway. Curr Med Sci. 2020;40(2):307-312. doi:10.1007/s11596-020-2178-0

24. Zhong S, Guo H, Wang H, et al. Apelin-13 alleviated cardiac fibrosis via inhibiting the PI3K/Akt pathway to attenuate oxidative stress in rats with myocardial infarction-induced heart failure. Biosci Rep. 2020;40(4):4. doi:10.1042/BSR20200040 
25. Hou N, Wen Y, Yuan X, et al. Activation of Yap1/Taz signaling in ischemic heart disease and dilated cardiomyopathy. Exp Mol Pathol. 2017;103(3):267-275. doi:10.1016/j.yexmp.2017.11.006

26. Li C, Wang J, Wang Q, et al. Qishen granules inhibit myocardial inflammation injury through regulating arachidonic acid metabolism. Sci Rep. 2016;6(1):36949. doi:10.1038/srep36949

27. Leach JP, Heallen T, Zhang M, et al. Hippo pathway deficiency reverses systolic heart failure after infarction. Nature. 2017;550 (7675):260-264. doi:10.1038/nature24045

28. Magnusson PU, Dimberg A, Mellberg S, et al. FGFR-1 regulates angiogenesis through cytokines interleukin-4 and pleiotrophin. Blood. 2007;110(13):4214-4222. doi:10.1182/blood-2007-01-067314

29. Perez-Pinera P, Chang Y, Deuel TF. Pleiotrophin, a multifunctional tumor promoter through induction of tumor angiogenesis, remodeling of the tumor microenvironment, and activation of stromal fibroblasts. Cell Cycle. 2007;6(23):2877-2883. doi:10.4161/cc.6.23.5090

30. Liu S, Shen M, Hsu EC, et al. Discovery of PTN as a serum-based biomarker of pro-metastatic prostate cancer. Br J Cancer. 2021;124 (5):896-900. doi:10.1038/s41416-020-01200-0

31. Hamma-Kourbali Y, Bermek O, Bernard-Pierrot I, et al. The synthetic peptide $\mathrm{P} 111-136$ derived from the $\mathrm{C}$-terminal domain of heparin affin regulatory peptide inhibits tumour growth of prostate cancer PC-3 cells. BMC Cancer. 2011;11(1):212. doi:10.1186/1471-2407-11-212

32. Xi G, Demambro VE, D'Costa $\mathrm{S}$, et al. Estrogen stimulation of pleiotrophin enhances osteoblast differentiation and maintains bone mass in IGFBP-2 null mice. Endocrinology. 2020;161(4). doi:10.1210/endocr/bqz007

33. Nikitovic D, Berdiaki A, Zafiropoulos A, et al. Lumican expression is positively correlated with the differentiation and negatively with the growth of human osteosarcoma cells. FEBS J. 2008;275(2):350-361. doi:10.1111/j.1742-4658.2007.06205.x

34. Radwanska A, Litwin M, Nowak D, et al. Overexpression of lumican affects the migration of human colon cancer cells through up-regulation of gelsolin and filamentous actin reorganization. Exp Cell Res. 2012;318(18):2312-2323. doi:10.1016/j.yexcr.2012.07.005

35. Nikitovic D, Chalkiadaki G, Berdiaki A, et al. Lumican regulates osteosarcoma cell adhesion by modulating TGF $\beta 2$ activity. Int $J$ Biochem Cell Biol. 2011;43(6):928-935. doi:10.1016/j. biocel.2011.03.008

36. Nagasawa A, Kubota R, Imamura Y, et al. Cloning of the cDNA for a new member of the immunoglobulin superfamily (ISLR) containing leucine-rich repeat (LRR). Genomics. 1997;44(3):273-279. doi:10.1006/geno.1997.4889

37. Xu J, Tang Y, Sheng X, et al. Secreted stromal protein ISLR promotes intestinal regeneration by suppressing epithelial Hippo signaling. EMBO J. 2020;39(7):e103255. doi:10.15252/embj.2019103255

38. Zhang K, Zhang Y, Gu L, et al. Islr regulates canonical Wnt signaling-mediated skeletal muscle regeneration by stabilizing Dishevelled-2 and preventing autophagy. Nat Commun. 2018;9 (1):5129. doi:10.1038/s41467-018-07638-4

39. Hara A, Kobayashi H, Asai N, et al. Roles of the mesenchymal stromal/stem cell marker meflin in cardiac tissue repair and the development of diastolic dysfunction. Circ Res. 2019;125 (4):414-430. doi:10.1161/CIRCRESAHA.119.314806

International Journal of General Medicine

\section{Publish your work in this journal}

The International Journal of General Medicine is an international, peer-reviewed open-access journal that focuses on general and internal medicine, pathogenesis, epidemiology, diagnosis, monitoring and treatment protocols. The journal is characterized by the rapid reporting of reviews, original research and clinical studies

Submit your manuscript here: https://www.dovepress.com/international-journal-of-general-medicine-journal
40. Polley A, Khanam R, Sengupta A, et al. Asporin reduces adult aortic valve interstitial cell mineralization induced by osteogenic media and wnt signaling manipulation in vitro. Int $J$ Cell Biol. 2020;2020:2045969. doi:10.1155/2020/2045969

41. Sasaki Y, Takagane K, Konno T, et al. Expression of asporin reprograms cancer cells to acquire resistance to oxidative stress. Cancer Sci. 2021;112(3):1251-1261. doi:10.1111/cas.14794

42. Castellana B, Escuin D, Peiró G, et al. ASPN and GJB2 are implicated in the mechanisms of invasion of ductal breast carcinomas. $J$ Cancer. 2012;3:175-183. doi:10.7150/jca.4120

43. Simkova D, Kharaishvili G, Korinkova G, et al. The dual role of asporin in breast cancer progression. Oncotarget. 2016;7 (32):52045-52060. doi:10.18632/oncotarget.10471

44. Wang HB, Huang R, Yang K, et al. Identification of differentially expressed genes and preliminary validations in cardiac pathological remodeling induced by transverse aortic constriction. Int J Mol Med. 2019;44(4):1447-1461. doi:10.3892/ijmm.2019.4291

45. Li XL, Yu F, Li BY, et al. The protective effects of grape seed procyanidin B2 against asporin mediates glycated low-density lipoprotein induced-cardiomyocyte apoptosis and fibrosis. Cell Biol Int. 2019. doi:10.1002/cbin.11229

46. Lu A, Kamkar M, Chu C, et al. Direct and indirect suppression of Scn5a gene expression mediates cardiac $\mathrm{Na}+$ channel inhibition by Wnt signalling. Can J Cardiol. 2020;36(4):564-576. doi:10.1016/j. cjca.2019.09.019

47. He J, Cai Y, Luo LM, et al. Expression of Wnt and NCX1 and its correlation with cardiomyocyte apoptosis in mouse with myocardial hypertrophy. Asian Pac J Trop Med. 2015;8(11):930-936. doi:10.1016/j.apjtm.2015.10.002

48. Yatabe J, Sanada H, Yatabe MS, et al. Angiotensin II type 1 receptor blocker attenuates the activation of ERK and NADPH oxidase by mechanical strain in mesangial cells in the absence of angiotensin II. Am J Physiol Renal Physiol. 2009;296(5):F1052-1060. doi:10.1152/ ajprenal.00580.2007

49. Kakhi S, Phanish MK, Anderson L. Dilated cardiomyopathy in an adult renal transplant recipient: recovery upon tacrolimus to sirolimus switch: a case report. Transplant Proc. 2020;52(9):2758-2761. doi:10.1016/j.transproceed.2020.06.011

50. Gao G, Chen W, Yan M, et al. Rapamycin regulates the balance between cardiomyocyte apoptosis and autophagy in chronic heart failure by inhibiting mTOR signaling. Int J Mol Med. 2020;45 (1):195-209. doi:10.3892/ijmm.2019.4407

51. $\mathrm{Xu} \mathrm{CN}$, Kong $\mathrm{LH}$, Ding $\mathrm{P}$, et al. Melatonin ameliorates pressure overload-induced cardiac hypertrophy by attenuating Atg5-dependent autophagy and activating the Akt/mTOR pathway. Biochim Biophys Acta Mol Basis Dis. 2020;1866(10):165848. doi:10.1016/j.bbadis.2020.165848

52. $\mathrm{Li} \mathrm{Y,} \mathrm{Lu} \mathrm{H,} \mathrm{Xu} \mathrm{W,} \mathrm{et} \mathrm{al.} \mathrm{Apelin} \mathrm{ameliorated} \mathrm{acute} \mathrm{heart} \mathrm{failure} \mathrm{via}$ inhibiting endoplasmic reticulum stress in rabbits. Amino Acids. 2021;53(3):417-427. doi:10.1007/s00726-021-02955-3 across all disease areas. The manuscript management system is completely online and includes a very quick and fair peer-review system, which is all easy to use. Visit http://www.dovepress.com/ testimonials.php to read real quotes from published authors. 